Revue internationale d'éducation de Sèvres

34 | décembre 2003

La formation professionnelle initiale: une question de société

\title{
Russie : un système éducatif en rénovation
}

Didier Paris

\section{CpenEdition}

Journals

Édition électronique

URL : https://journals.openedition.org/ries/1751

DOI : $10.4000 /$ ries. 1751

ISSN : 2261-4265

Éditeur

France Education international

Édition imprimée

Date de publication : 1 décembre 2003

Pagination : 153-162

ISBN : 978-2-8542-0558-9

ISSN : $1254-4590$

Référence électronique

Didier Paris, «Russie : un système éducatif en rénovation », Revue internationale d'éducation de Sèvres

[En ligne], 34 | décembre 2003, mis en ligne le 23 novembre 2011, consulté le 06 juillet 2021. URL

http://journals.openedition.org/ries/1751 ; DOI : https://doi.org/10.4000/ries.1751

Ce document a été généré automatiquement le 6 juillet 2021

(c) Tous droits réservés 


\title{
Russie : un système éducatif en rénovation
}

\author{
Didier Paris
}

1 Le système russe d'éducation, confronté à des transformations majeures de l'économie et de la société tout entières, est l'enjeu de très importants changements. Un programme de "rénovation » est engagé par le ministère fédéral de l'Éducation. Sa mise en œuvre donne lieu à des débats et controverses passionnés tant auprès des enseignants et des élèves que dans l'opinion publique. Dans un cadre où la centralisation de la période soviétique tend à prendre des formes nouvelles, le désir de rénover l'école apparaît dans des initiatives régionales et locales qui deviennent parfois des moteurs d'innovation.

\section{Les héritages successifs}

2 Le système d'éducation soviétique a été élaboré dans le courant des années vingt du Xx siècle. S'inspirant des mouvements pédagogiques progressistes de l'époque, il installait, à l'instar des pays scandinaves, une école unique où les enfants étudiaient ensemble la même chose du début à la fin de la scolarité. Les établissements scolaires russes s'appellent d'ailleurs simplement « écoles ${ }^{1}$.

3 L'État soviétique a cherché d'abord à assurer par l'éducation la promotion sociale des ouvriers et des paysans tout en répondant aux besoins de formation créés par l'économie à chaque époque historique. Progressivement, à mesure que se sont installées aux postes de pouvoir les générations instruites, elles ont fait en sorte que l'enseignement leur permette de reproduire la nouvelle élite.

4 La gestion de l'éducation, étroitement centralisée et contrôlée, échappait souvent aux ministères de l'Éducation, le pouvoir réel étant assuré par le parti, le Gosplan, les ministères techniques et les combinats ${ }^{2}$.

5 Les années quatre-vingt-dix ont vu à la fois l'effondrement des dépenses publiques, particulièrement pour l'éducation, et une décentralisation de la décision: le 
financement et la gestion de l'éducation sont revenus aux collectivités locales, parfois même aux établissements. Cela s'est accompagné d'une dérégulation permettant la recherche de capitaux privés, et l'apparition d'établissements privés.

6 La Russie a connu en même temps une chute impressionnante de sa natalité. Pendant la décennie 90-2000, des classes d'âge de moins en moins nombreuses sont arrivées dans les écoles. La tendance va concerner à présent l'enseignement supérieur³ .

7 Le système de la période soviétique se distinguait par son monolithisme et son uniformité, ne tolérant pas officiellement de variantes d'une école à l'autre.

Une « différenciation » croissante est apparue entre les établissements : la majorité des écoles " ordinaires", pratiquement sans moyens autres que le bénévolat, assurent un minimum en termes d'innovation, ou tout simplement de fonctionnement. D'autres, souvent sous la tutelle d'universités liées à des sponsors, deviennent des écoles « d'élite » aux noms prestigieux et se réorientent de préférence vers les formations les plus demandées par les familles de "nouveaux Russes $»^{4}$ : gestion, management, publicité, métiers de l'entreprise et de la finance, droit, pour lesquelles se développe un véritable marché. L'élitisme autrefois réprouvé est désormais une valeur positive.

C'est dans le contexte des retombées de la crise économique et monétaire d'août 1998 et d'une apathie généralisée de l'initiative en éducation que fut nommé ministre le recteur Filippov ${ }^{5}$ candidat de consensus dont on n'attendait pas a priori de bouleversements. Sa désignation fut approuvée par le groupe communiste de la Douma d'État et par l'union des recteurs d'université. Recteur entouré de recteurs, il a permis aux universités d'impulser les réformes et d'asseoir leur autorité, sinon leur mainmise, sur l'enseignement scolaire, les examens, et même les contenus.

Le ministère de l'Éducation reprend alors la main et rassemble les outils de pilotage des changements pour tenter de définir une politique éducative se fixant un objectif de " qualité ", se référant aux "standards " mondiaux pour les deux ou trois décennies à venir. Le ministre est aussi le premier à avoir permis l'introduction d'autres mesures «à l'occidentale » dans les écoles comme dans l'enseignement supérieur : création de MBA pour former des hommes d'affaires, introduction de technologies de formation à distance, de l'informatique et d'Internet à l'école. Ce " progressisme » est également à mettre à l'actif du recteur de la nouvelle Haute école d'économie, M. Kouzminov, son plus proche conseiller.

11 Le ministère de l'Éducation s'est de fait transformé en un comité de pilotage des réformes et de l'innovation, rassemblant les plus imaginatifs et les plus efficaces représentants des recteurs, des directeurs d'écoles, et des spécialistes des sciences de l'éducation.

\section{Structure et contenus : des décisions hardies}

12 Un premier chantier est ouvert dès 1999 sur l'allongement de la scolarité à douze ans. L'idée ne reçoit pas un accueil très enthousiaste du public, poussé notamment par les critiques des médias assez souvent mal informés.

13 Les spécialistes de l'académie russe de l'éducation (russkaya akademia obrazovaniya), chargés notamment des dossiers du renouvellement des programmes et de la mise en place de filières pour les dernières classes, ont préparé de solides projets. Cette réforme prévoit la modernisation des objectifs et des contenus, la création d'options, la 
spécialisation des établissements. Dans ce sens, on peut considérer qu'elle a été pour l'essentiel appliquée.

Un second chantier est lancé en 2001. Il reprend l'essentiel des objectifs précédents, le changement d'orientation (ou tout au moins présenté comme tel) résidant dans la création d'un examen unique national de fin d'études scolaires.

On parle officiellement de « rénovation » ou de « modernisation " pour éviter le terme de « réforme », soulignant la nécessaire continuité de l'éducation et même l'absence de rupture brutale par rapport aux périodes précédentes, qui avaient également leurs qualités et leurs résultats. C'est aussi certainement ce que pense la majorité des adultes russes, souvent attachés au souvenir de l'école de leur jeunesse.

\section{Allongement de la scolarité dans un contexte d'effondrement de la natalité}

Les Russes pensent généralement que la santé des enfants est de plus en plus en danger $\mathrm{du}$ fait de la surcharge scolaire. Ce souci, tout comme la référence aux recommandations des médecins, ne constituent pas un exercice de style. Il s'agit d'un réel problème : la pratique de copieux devoirs à la maison est la règle générale. Mais surtout, de nombreux parents ont recours massivement à des cours particuliers dispensés par le professeur habituel ou par d'autres. Car dans les conditions actuelles de la "transition ", l'accès aux études universitaires et aux carrières intéressantes est conditionné par la qualité du dossier scolaire ainsi que par des concours d'accès aux universités parfois très difficiles. Allonger la scolarité permet ainsi de réduire programmes et horaires. La stratégie d'allongement des études répond aussi et surtout à la progression du déficit d'élèves des classes d'âge scolaire. L'effectif global d'élèves sera passé de vingt-et-un à treize millions d'ici 2011...

Dès 2000, la durée de la scolarité obligatoire est passée à dix ans (contre huit auparavant) et le cycle complet d'études à douze ans (contre dix). La durée des études primaires et secondaires (enseignement "moyen ») est en 2003 de onze ans pour $60 \%$ des élèves, et de dix ans pour les $40 \%$ restants, les classes de $12^{\mathrm{e}}$ restant très peu nombreuses. L'inversion de ces pourcentages a été réalisée en deux ans par allongement d'un an de l'enseignement primaire, qui débute à l'âge de six ans au lieu de sept précédemment. L'orientation vers l'enseignement professionnel et technologique pratiquée à l'issue de la $9^{\mathrm{e}}$ classe est retardée d'une année.

18 Cette politique intègre la recommandation du Conseil de l'Europe de 1992 d'allonger la scolarité à douze ou quatorze ans pour atteindre un niveau de fin d'études à dix-huit ou dix-neuf ans semblable à celui des pays les plus développés.

\section{Révision des objectifs de l'enseignement moyen}

19 Les textes officiels citent les priorités : « adapter l'enseignement à la nouvelle société, rompre avec l'école soviétique, former une personnalité harmonieusement développée capable de concrétiser son potentiel créatif dans le contexte de l'économie de marché, dans sa vie privée, mais aussi pour le bien de la société $»^{6}$.

Un certain nombre de recommandations pour une approche plus humaniste font l'objet d'une particulière insistance : approche culturelle et sociale, enseignement des sciences 
sociales et des langues étrangères depuis un âge précoce, développement des capacités d'autonomie et de jugement, utilisation des nouvelles technologies, orientation, ambition professionnelle, renforcement de l'attachement aux valeurs morales, au patriotisme, à la spiritualité.

Un débat s'est instauré sur l'opportunité d'introduire un enseignement de la religion orthodoxe, «base incontournable de la civilisation russe ». Le patriarcat exerce dans ce débat, comme auprès des responsables politiques, un rôle de premier plan. Tous ces aspects font en fait réponse à une très forte demande sociale.

Les critiques adressées à l'école portent souvent sur le manque de considération de l'individu, l'injustice des notes, la priorité accordée aux savoirs mémorisés dont le contrôle n'exclut pas la tricherie, la médiocrité et l'indifférence de certains enseignants, le favoritisme et même la corruption. On réfléchit à la vie scolaire, à l'autogestion, à la citoyenneté à l'école.

Doter les jeunes d'outils leur permettant de s'informer de façon autonome sur le monde et de se former une opinion ou un jugement à partir d'informations non contrôlées, voilà qui constitue une réelle innovation par rapport aux traditions héritées du système précédent où l'étranger, peu présent dans le cursus scolaire et a priori peu valorisé était abordé à travers un prisme idéologique qui excluait en particulier toute utilisation de document authentique.

\section{Différenciation ${ }^{7}$ des études et des parcours}

La scolarité (douze ou onze années) est divisée en trois « degrés »:

- le premier ( $1^{\text {re }}$ à $4^{\mathrm{e}}$ classe, de six à dix ans) assure un développement de la personnalité, de l'intelligence et de la motivation à apprendre. L'enseignement d'une langue étrangère et des technologies de l'information devront débuter en $2^{\mathrm{e}}$ classe $^{8}$;

- dans le deuxième degré ( $5^{\mathrm{e}}$ à $10^{\mathrm{e}}$ classe) l'enseignement général est organisé par « blocs », et vise à une orientation ;

- dans le troisième degré, l'allongement de la scolarité s'accompagne de la création de filières (les deux dernières classes), pour permettre d'élargir et de démocratiser l'accès à la formation professionnelle universitaire moyenne et supérieure, rendue aussi plus accessible $\mathrm{du}$ fait de la baisse démographique. Ces classes se rapprochent des sections L, S, ES, T, etc., des classes de première et terminale en France. On y dispense un enseignement général, plus un enseignement orienté vers les aptitudes, les dispositions, les goûts et intérêts des élèves.

Les connaissances générales minimales pour toutes les filières sont clairement définies mais la façon dont les enseignements sont dispensés est laissée au choix de chaque établissement: en classe ou à distance, le jour ou le soir et toutes les formes non conventionnelles d'apprentissage sont encouragées, jusqu'à composer pour chaque élève un parcours personnel comprenant des matières de base, de spécialité, et d'approfondissement, intégrant la recherche personnelle.

L'introduction d'une telle « différenciation » constitue une sorte de révolution dans un pays où les élèves avaient toujours tous étudié les mêmes sujets du début à la fin de la scolarité et où l'orientation était subordonnée aux besoins de l'économie.

L'idée de l'école de douze ans n'est plus aujourd'hui réellement mise en avant par le ministère, d'autant que la Douma, ayant jugé que l'allongement de la scolarité est du ressort du législateur, s'est déclarée en première lecture pour le maintien des études à 
onze ans. Le débat se poursuit. Le principe de la différenciation semble, en revanche, irréversible.

\section{Différenciation des établissements et recomposition de la décision en éducation}

Le "plan d'études de base » introduit officiellement le principe de la différenciation : les établissements scolaires peuvent se spécialiser en choisissant parmi des " variantes ", les autorités décentralisées apportant leur part locale de décision. Cette flexibilité tranche avec la situation monolithique du système précédent.

Ces dispositifs ont ouvertement un objectif élitiste, ce qui ne choque personne en Russie. Compétitions, concours scolaires, olympiades, médailles d'or et d'argent pour les meilleurs élèves ont toujours existé, d'ailleurs".

Pour se différencier, les écoles peuvent choisir entre plusieurs options.

\section{Les classes spécialisées}

31 Dans les classes "spécialisées " (ou spetschkoly, qui existaient déjà en nombre plus réduit), l'enseignement d'une ou plusieurs matières est renforcé à partir d'un niveau donné. Ce peuvent être les mathématiques, les sciences, le sport, la musique, une ou plusieurs langues, la littérature... Ces classes, où les enfants entrent après une sélection, coexistent alors avec des classes ordinaires dans la même école.

\section{Les classes «profilées » ou " gymnasiques »}

Les classes dites "profilées » ou "gymnasiques " (gymnasitcheskyé) mènent vers une filière d'enseignement supérieur. On voit se multiplier ces sortes de classes préparatoires vers telle ou telle université, où interviennent des universitaires. Une convention est alors passée entre les établissements secondaire et supérieur. Lorsque l'établissement entier est ainsi profilé vers un ou plusieurs établissements d'enseignement supérieur, il peut prendre le nom prestigieux de « lycée » (litsiei) - de la huitième classe à la dizième ou onzième - ou "gymnase» (gymnasium) - de la cinquième ou septième classe à la onzième. On y étudie en général deux langues, et des matières en approfondissement.

\section{Les collèges}

Le terme de « kolledj » est réservé aux établissements professionnels et technologiques $(\text { PTU })^{10}$ qui contractent avec des universités, des instituts et surtout avec des entreprises. Certains dépendent directement des entreprises ou d'autres ministères hors Éducation nationale.

\section{Les autres établissements}

De nouveaux types d'établissement apparaissent, comme les écoles pour surdoués, les écoles bilingues (anglais), et tous les types imaginables d'écoles novatrices du secteur privé. 
d'enseignement et d'encadrement, le suivi minutieux du développement personnel de leurs élèves, l'orientation, l'innovation et la création. Avec la différenciation et les filières, la mobilité, la flexibilité, ils affrontent la concurrence. Ces nouveautés ne sont pas pour l'instant accompagnées d'une annonce d'effort de formation continue. Or le problème des enseignants réside dans le très bas niveau de leurs salaires. Le salaire moyen est passé de 27 euros en 1999 à 55 euros en 2001. Il doit atteindre 70 à 80 euros en 2003, soit $55 \%$ du salaire moyen national. Il n'a dépassé le minimum vital pour la population active qu'en 2001. Cet état de fait permet de comprendre : les professeurs du secondaire; le vieillissement alarmant du corps enseignant: la moitié des instituteurs ont plus de quinze ans d'ancienneté. Moins de $30 \%$ des professeurs ont une ancienneté inférieure à dix ans. Les retraités constituent $10 \%$ des professeurs.

La Douma d'État a voté un texte, non ratifié par l'exécutif, dispensant du service militaire (deux ans) les jeunes qui acceptent d'enseigner. Cela permettrait de rajeunir et de masculiniser la profession. De nombreux jeunes enseignants dynamiques ont quitté le métier. Les enseignants complètent leur revenu en acceptant plusieurs postes dans deux ou plusieurs établissements, en donnant des cours du soir et des leçons particulières. Leur charge hebdomadaire moyenne se situe entre quarante et cinquante heures de cours, ce qui nuit à la qualité de l'enseignement.

41 La trop lente amélioration de leur statut social permet donc de comprendre leur hostilité générale à une réforme décidée sans les consulter mais qui, en tout état de cause, ne peut se faire sans eux.

\section{Évaluation, certification et sélection}

Les élèves reçoivent jusqu'à présent une attestation de fin d'études faisant suite à un examen passé dans l'école et corrigé par leurs professeurs. Les notes vont de 1 à 5 . Les meilleurs élèves sont récompensés par des médailles d'or et d'argent. Pour entrer dans un établissement d'études supérieures, on doit réussir un concours. 

écennie précédente et mis en place de façon volontariste des changements de structure pour les décennies à venir. De vraies réformes ont été engagées de façon irréversible.

Le nouveau système prévoit un examen unique d'État pour toute la fédération, l'EGE $E^{11}$, dont les résultats donneront accès de façon différenciée aux universités. La note finale, sur plusieurs centaines de points, additionne les notes des différentes matières : russe et mathématiques pour tous ainsi que trois matières choisies dotées de coefficients.

4 Un dispositif complexe attribue des points aux étudiants selon leurs résultats. À ces points correspondent des financements par l'État aux universités choisies par les lauréats. Cela permet de lier les ressources des universités au niveau des étudiants qu'elles recrutent.

5 Les cycles de préparation aux concours d'entrée, ressource importante des universités et de leurs enseignants, ne sont plus nécessaires. La réforme se donne d'ailleurs pour premier objectif de mettre fin à la corruption, « honte nationale » selon les paroles du ministre, qui concernerait, selon certains sociologues, jusqu'à $20 \%$ des lauréats des écoles. Il s'agit de garantir l'égalité d'accès pour tous aux universités. L'examen unique permettra aussi d'évaluer et comparer les régions, les écoles, et même les enseignants.

La moitié à peu près des élèves de Russie concernés, soit 650000 élèves, a passé cette année l'examen unique dans quarante-sept régions. Il en est à sa troisième année d'existence et doit être généralisé en 2005.

L'implantation du EGE soulève dans l'opinion publique, chez les élèves et leurs parents et surtout chez les enseignants, des réactions parfois violemment négatives.

8 La nécessité de rénover le système, de même que les principes généraux des réformes, font l'objet d'un large consensus. Ce sont la conception et la rédaction des épreuves qui concentrent les critiques.

9 Les professeurs ont unanimement souligné l'inadaptation des épreuves. Elles ne permettent pas, disent-ils, d'évaluer les connaissances ni les aptitudes. Tests et questionnaires remplacent l'ancienne dissertation de six heures, l'essai en langue étrangère, etc. Cela induit en confusion l'élève cultivé, habitué à la réflexion approfondie et à la synthèse. La forme du QCM, imitée des modèles anglo-saxons, est utilisée dans toutes les matières. L'examen est comparé à un jeu télévisé («Comment devenir millionnaire " est extrêmement apprécié en Russie). Le risque est de favoriser une pédagogie du bachotage au détriment de la culture, de la réflexion, et de l'esprit critique.

D'autre part les résultats, de l'aveu même du ministère, s'avèrent catastrophiques. Les notes, très basses dans certaines régions, ont du être remontées pour éviter des remous sociaux : 30 à $40 \%$ des étudiants « n'avaient pas le niveau » requis par les «standards ». L'examen unique révèlerait, en fait, les insuffisances qualitatives du système d'enseignement dans son ensemble...

\section{Quelles décisions pour l'école}

Dès 2000, les responsables éducatifs russes ont statué sur les tendances nouvelles de la 
Conclusions de l'évaluation globale menée en 2003

Le ministère de l'Éducation a résolu de se doter d'instruments fiables pour définir et
piloter les réformes. Les résultats de Penquête PISA $2000(1)^{1}$ avaient déj four piloter lests refermes. tes remparaison avec le reste do nde au niveau des performanarn quelque Un rapport réalisé sous lautorité du ministère fédéral de l'Éducation évalue le système ulsse déduction avec les criteres internationaux Il s'agit de situer la Russie par rappo ter tendances mondales de developpement des systemes educatifis. La confrontation dynamique de statistiques démographiques et économiques et de donnéé

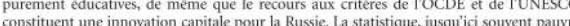
ou incohérente, de diffusion confidentielle, et encore parfois idéologique, devient un out pour les décideurs au service de la politique de reformes qui ils ont engages.

Chute de la population d'âge scolaire

La Russie se situe dans le troisième groupe (sur quatre) pour linindice de développement baisse brutale de la natalité dans les années quatre-vingt-dix a fait décroitre rapidement pour les 5-14 ans, et de $33 \%$ pour les $15-19$ ans dici 2010 , et respectivement de $38 \%$ of $47 \%$ d'ici 2015. Cela constitue un record mondial.

Scolarité trop courte

La Russie se distingue des autres pays par le degré de scolarisation aux différents âges. Si, offiellement, mas sept et mâme huit ans pour ples de $10 \%$ des enfants. Les jardins d'enfants russes, qui n'ont pas a proprement parler d'objectifs pedagogiques, ne sont pas des etablissements scolaires au sens ou fentend UNESCO. La duree des etudes es mons longue que dans har plupart des pays. Pour les eleves do est déà scolarisée dans l'enseignement supérieur, qui « déborde * donc largement sur le secondaire.

Absence de formations supérieures courtes et hypertrophie des troisièmes cycles

es formations supérieures courtes (inférieures à cinq ans) n'existent pour ainsi dire pas et de lenseignement professionnel moyen. Ensuite, les effectifs deppassent ceux des pays lo plus riches en ce qui concerne le nombre de doctorants et de docteurs ( $3^{\mathrm{e}}$ cycle).

Un corps enseignant pléthorique et féminisé

Comparé aux autres pays, le nombre d'éleves ou d'étudiants par enseignant est très faible,
plus faible que dans la plupart des pays les plus riches, particulièrement dans Penseignement superieur. La Russie apparait comme un pays denseignants trop nombreux et max payés. La féminisation du corps enseignant $y$ est plus poussee : $7 \%$ tous nive

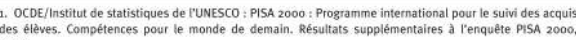

Charge scolaire des élèves au-dessous de la moyenne

Lopinion publique russe est convaincue que la surcharge de travail des écoliers, confinan au surmenage, menace leur sante. Lallongement de la scolarisation a douze ans prevue par le ministre sappuyait d'ailleurs sur cet argument. L'enquête montre qu'il n'en est rien ; les horaires de cours ressemblent a ceux des autres pays et sont méme bien inferieurs pou certains niveaux. Les jeunes Russes ont plus d heures de lecture, d ecriture et de litterature, un peu plus de sciences, mais moins de sciences sociales, et nettement moins d'éducation artistique et d'éducation physique que les autres.

Contrôle étatique mais financement régional

La Russie est l'un des pays dont le financement de l'enseignement primaire, secondaire et professionnel non supérieur est le plus largement assuré par les autorités régionales a locales $(93 \%)$, consequence des transferts de gestion des secteurs non rentables dans les années quatre-vingt-dix. L.es dépenses de l'enseignement supérieur restent assurées par le budget d'État $(81 \%)$ comme dans la plupart des pays.

Financement exceptionnellement faible de l'éducation

Par rapport à son PIB, la Russie a un niveau « extraordinairement bas " de financement de l'éducation : 4,1\%. Très peu de pays (Indonésie, Chine) sont au-dessous. Ce déficit de financement est particulièrement accentué dans l'enseignement scolaire primaire et secondaire (ecoles moyennes, ecoles professionnelles et techniques). Rapporté a la scolarisation réelle de la population, Tindice de financement par élève est l Iun des plus bas du

Plus le pinver le coût relatif par élève (ou étudiant) s'accroît. La presta place ce pays devant de

Ce très bas niveau de dépenses d'éducation n'est pas liế à un bas niveau des dépensee La Russie est parmi les pour corollaire une politique éducative très peu active de lEtat. principalement l'enseimement primaire et secondire. Il faut y ajouter l'inefficacité des moyens accordés à l'enseignement préscolaire (les jardins d'enfants n’étant que des " garderies" au regard des criteres internationaux).

On pense généralement en Russie que, malgré des revenus par habitant modestes, le pays a un système d'éducation correspondant au niveau des pays les plus riches. L'analyse des indicateurs de qualité, pratiquée à l'aide des instruments de mesure internationalement reconnus, vient démentir ce jugement optimiste :

Les résultats de la Russie sont inférieurs à la moyenne des pays de revenu comparable et inférieurs à ceux de nombre des pays les plus pauvres en ce qui concerne le financement de l'éducation.

Paradoxalement, la crise démographique vient en quelque sorte atténuer les conséquences négatives de cet état de fait.

52 L'éducation repose en Russie sur des traditions solides et des réussites incontestables. Tous y sont attachés et il n'est pas question d'en faire table rase. Un consensus se manifeste quoi qu'il en soit sur la nécessité de moderniser l'école. 

sur celui des bouleversements socio-économiques récents. Les points noirs de l'école sont identifiés et de nouvelles orientations sont déterminées :

- l'adaptation à une société démocratique, qui exige un changement d'état d'esprit dans les objectifs : ouverture, esprit critique, expression des opinions, épanouissement personnel, créativité ;

- l'adaptation à l'économie de marché, qui nécessite la libération des ambitions, une refonte totale de l'orientation scolaire et universitaire, un nouveau rapport à l'économie et à l'argent, la valorisation des élites.

L'exigence de qualité impose l'allongement de la scolarité, la mise en place de dispositifs sérieux d'évaluation des résultats mais aussi du système et de ses acteurs ainsi que l'utilisation de nouvelles technologies.

L'ouverture internationale de la Russie la place en position de contact ou de concurrence avec d'autres pays. Les jeunes Russes devront connaître des langues étrangères, étudier à l'étranger, voyager. L'entrée de la Russie dans le processus de Bologne pour la mise en phase des cursus et des diplômes universitaires est désormais lancée.

Quoi qu'il en soit, le système éducatif russe ne pourra être à l'égal des systèmes des pays les plus avancés sans un travail de fond sur les financements.

Opinions, tendances, pressions, lobbies s'expriment sur les mesures à prendre, dans un formidable bouillonnement d'idées, dans les média et sur les forums. Les partisans de la voie « libérale », préconisée par la Banque mondiale, fort active en Russie, tirent le pays vers les solutions à l'anglo-saxonne, tandis que la tradition nationale pousse au maintien d'un système centralisé sous contrôle de l'État.

\section{BIBLIOGRAPHIE}

BAJOMI I. et DEROUET J.-L. (dir.) : La grande récréation : la décentralisation de l'éducation dans six pays autrefois communistes, INRP, 2002.

VINOKUR A. (dir.) : Les transformations du système éducatif de la Fédération de Russie, UNESCO, 2001.

SIGMAN C. : «Quel système éducatif pour la Russie : l'affrontement de deux modèles », Le courrier des pays de l'Est, $\mathrm{n}^{\circ}$ 1007, août 2000, pp. 32-44.

L'enseignement en Russie 2001 : recueil statistique d'information et d'analyse, Ministère de l'éducation de la Fédération de Russie.

L'enseignement en Russie 2002 : recueil statistique d'information et d'analyse, Ministère de l'éducation de la Fédération de Russie.

POLETAYEV A.V., AGRANOVITCH M.L., JAROVA L.N. : Rossiisskoyé obrazovanié v kontekste mejdunarodnykh pokazateleï; Sopostavitelnyi doklad (L'enseignement russe dans le contexte des indicateurs internationaux ; Rapport comparatif), Aspekt Press, 2003. 
Compétences pour le monde de demain : résultats supplémentaires à l'enquête PISA 2000, OCDE - Institut d'études statistiques de l'UNESCO, 2003.

\section{NOTES}

1. La distinction française «école ", « collège », « lycée » n'a pas de pertinence en Russie. On utilisera donc le terme russe d' "école».

2. Combinat : structure régionale de production industrielle à forte concentration.

3. La baisse de la population d'âge scolaire depuis 1990 a touché de nombreux pays d'Europe (Suisse, Allemagne, France...). Elle atteint plus encore les pays d'Europe centrale et orientale. La baisse pour la tranche d'âge 5-19 ans atteint un chiffre record en Russie. Les prévisions annoncent pour 2010 une diminution d'un tiers (et pour 2015 de la moitié) des effectifs scolaires.

4. Appellation devenue courante et désignant les «nouveaux riches » récemment apparus en Russie.

5. En Russie, le titre de recteur désigne le président d'une université. Pour l'équivalent d'un recteur d'académie, on parle de directeur régional de l'éducation, parfois de ministre (régional) de l'éducation.

6. Documents normatifs, directives du ministère fédéral de l'Éducation, 2001.

7. Le terme de «différenciation " s'applique à l'introduction de différences entre les individus (choix, options, filières) mais aussi entre les classes ou les établissements. Il reflète un souci nouveau de la personnalité de chacun, qui va à l'encontre du système soviétique réputé uniforme pour tous et ne prenait pas en compte les individus, mais les besoins de la société.

8. L'enseignement d'une langue étrangère en $2^{\mathrm{e}}$ classe est programmé pour la rentrée 2006.

9. Les valeurs positives ou négatives des notions d'égalité, de sélection, d'élitisme, pourraient donner lieu à une étude interculturelle comparative des bases de l'éducation entre la France et la Russie...

10. Professionalno-Tekhnitcheskyé oUtchilisché : établissements professionnels et techniques.

11. EGE, yEdiniy Gosudarstvenniy Egzamen : examen unique d'État.

\section{INDEX}

Index géographique : Russie

\section{AUTEUR}

\section{DIDIER PARIS}

Attaché de coopération pour le français près l'ambassade de France en Russie 